\title{
Algorithm of Digital Watermark Based on Wavelet
}

\author{
Siyu Lai \\ Department of Medical Imaging \\ North Sichuan Medical College \\ NanChong, China \\ 1sy791211@126.com
}

\author{
Juan Wang* \\ College of Computer Science \\ China West Normal University \\ NanChong, China \\ *the corresponding author \\ wjuan0712@126.com
}

\begin{abstract}
Analyze a human visual model based wavelet domain digital watermark algorithm and improved it. First conduct multi-level wavelet decomposition on original image and modify medium frequency coefficient, and then adapt the algorithm to embed the watermark into the source image. The experiment shows that the watermark embedded image enjoys better imperceptibility and robustness to JPEG compression, add gaussian noise, contrast enhancement e.t. traditional image processing operations.
\end{abstract}

Keywords-digital watermarking; human visual system; discrete wavelet transform; algorithm

\section{INTRODUCTION}

The swift development of multimedia and Internet enable the communication and transmission of images and videos and other multimedia information become easier and quicker. However, the replicability, modifiability and ease of dissemination of digital media information raised new challenge to copyright protection and information security of digital products. The pressing requirement to digital products protection and information security created digital watermarking technology a hot topic in the fields of multimedia information security researchers $[1,2]$. Digital Watermarking technology refers to the way that used to embedding latent watermarking in digital multimedia data with signal processing method and these markings are usually invisible, it can only be extract through dedicated detectors or reader unit. The technology can be break into two categories in view of the implementation process [3] (i.e. spatial and transform domain algorithm). The key point of spatial domain algorithms is embedding watermarking directly into the spatial host media, this method is the main modus of early digital watermarking implement [4] and there are more active researches in recent years. Spatial algorithms typically enjoy fast speed, but poor robustness generally and the size of watermarking that to be embedding is usually restricted. Transform domain method refers to convert the presentation format of host media to transform domain first and then perform the watermarking embedding. This method has many good features like robustness, invisibility and so on; some algorithms also combine with the current image and video compression standard. In methods of this sort, the wavelet based multimedia watermarking technology is a relatively active research area [5] in recent years, especially with the JPEG2000[6] standard subsumed the wavelet transform, which make the researches on the areas are more meaningful.

Lewis and Knowles quantify wavelet coefficients to realize image compression and obtained the quantitative array self-adapting in the light of the sensitivity that human eye response to noise [8], which result in the establishment of wavelet based vision model. Barni e.t. improved the visual model and apply it to the digital watermark embedding process. Decompose the original image with 4 levels of wavelet and then modify the coefficients with medium or high frequency of wavelet in each level according to visual model to embed watermarking. After analysis the image with watermark, the statistic show us the watermarks are principal hide in place of rich texture, dim intensity and marginal area of images, which ensure the imperceptibility of the image. Guzman, too, utilized the vision model and the difference is that he selected only the medium and high frequency part of the first level of wavelet to embed into watermark. And conduct JPEG compression, add Gaussian noise, cropping, geometry rotation, enhance intensity and other attack test, which indicate that the algorithm enjoy excellent robustness to these attacks in addition to JPEG compression.

We divided watermark into two parts that are embedded into the third horizontal detailed and vertical detailed band respectively to adjust coefficient on the basis of human vision system. The updated algorithm possessed robustness while improved the imperceptibility of images.

\section{VISUAL MODEL OF WAVELET DOMAIN WATERMARKING ALGORITHM}

As a branch of harmonic analysis, wavelet theory provides a uniform framework for a variety of signal and image processing. Image signal can be separated into sub image signals that enjoy different spatial resolution, frequency and direction properties [7] with the help of wavelet multiple resolution decomposition.

Image could be divided up into 4 sub-bands, one of which is low frequency and the rest 3 are of medium and high frequency detailed sub-bands through discrete wavelet transform, each of them with a size of the $1 / 4$ of original image and the low frequency sub-band could be carried out subdivision once again. Figure 1 represents 4 -level wavelet decomposition diagrams $[8,9]$.

We define $\mathrm{I}_{3}^{3}$ as the sub-bard of level 1 wavelet at the direction of $\theta, l=0,1,2,3, \theta \in\{0,1,2\}$. 
The visual model of wavelet domain watermarking algorithm consists of the following three parts:

\begin{tabular}{|c|c|c|c|c|}
\hline $\mathrm{I}_{3}^{3}$ & $\mathrm{I}_{3}^{3}$ & \multirow{2}{*}{$I_{2}^{0}$} & \multirow{3}{*}{$\mathrm{I}_{3}^{3}$} & \multirow{4}{*}{$\mathrm{I}_{0}^{0}$} \\
\hline $\mathrm{I}_{3}^{2}$ & $\mathrm{I}_{3}$ & & & \\
\hline \multicolumn{2}{|c|}{$\mathrm{I}_{2}^{2}$} & $\mathrm{I}_{2}^{1}$ & & \\
\hline \multicolumn{3}{|c|}{$\mathrm{I}_{1}^{2}$} & $\mathrm{I}_{3}^{\prime}$ & \\
\hline \multicolumn{4}{|c|}{$\mathrm{I}_{0}^{2}$} & $I_{0}^{1}$ \\
\hline
\end{tabular}

Figure 1. 4 Level of wavelet decomposition

(1) Human eyes are insensitive to noise from image of different directions and levels with medium and high frequency, especially for those at $45^{\circ}$ direction. Suppose different levels $l(l=0,1,2,3)$, sub-bands have distinct noise covering factors recorded as $S(l, \theta)$, then $S(l, \theta)$ could be estimate by formula (1).

$$
S(l, \theta)=\left\{\begin{array}{ll}
\sqrt{2}, & \text { if } \theta=1 \\
1 \quad \text { otherwise }
\end{array}\right\} \times\left\{\begin{array}{ll}
1.00, & \text { If } 1=0 \\
0.32, & \text { If } 1=1 \\
0.16, & \text { If } 1=2 \\
0.10, & \text { If } 1=3
\end{array}\right\}
$$

(2) Human visual system enjoys different visual sensitivity to noise from different intensity domain of the images and is insensitive to noise of very dark or bright image fields. Given that the intensive factor of spatial point $(i, j)$ in level $l$ is $L(l, i, j)$, then $L(l, i, j)$ could be estimate by formula (2).

$$
L(l, i, j)=\frac{1}{256} I_{3}^{3}\left(1+\left[\frac{i}{2^{3-l}}\right], 1+\left[\frac{j}{2^{3-l}}\right]\right)
$$

(3) Human eyes are extremely insensitive to noise of image textural fields, that is to say, texture could cover up high intensive noise. Let $L(l, i, j)$ be the factor of texture effect, which could be characterized by the square of varied detailed sub images local mean and the variance of low frequency band, calculate by a $2 \times 2$ neighborhood at pixel $(i$, j)respectively just like formula (3) shows.

$$
\begin{aligned}
& T(l, i, j)=\sum_{k=0}^{3-l} \frac{1}{16^{k}} \sum_{\theta=0}^{2} \sum_{x=0}^{1} \sum_{y=0}^{1}\left[I_{k+1}^{\theta}\left(y+\frac{i}{2^{k}}, x+\frac{j}{2^{k}}\right)\right]^{2} \\
& * \operatorname{Var}\left\{I_{3}^{3}\left(1+y+\frac{i}{2^{3-l}}, 1+x+\frac{j}{2^{3-l}}\right)\right\} \quad x=0,1 y=0,1
\end{aligned}
$$

The above three formulas indicate that the visual model considering the varied frequencies of human visual system, the sensitivity of different direction and the cover effect of different intensive contrast, which provides excellent evidence to decide the watermark strength and location where to embed the watermark.

This article select coefficients is mainly refer to formula (2) and embed watermark into the darker fields of the image.

\section{IMBEDDING AND EXTRACTION OF WATERMARK}

\section{A. Watermark Embedding}

After watermark signal generated, map the embedded binary image to one dimensional vector $w m$ using line scanning method and $\mathrm{wm}$ is defined as

$$
w m=\{w m(i) \mid w m(i) \in\{0,1\}, 1 \leq i \leq p \times q\} \text { where }
$$

$N=p \times q$ is the size of image.

We break watermark embedding process into the following steps:

Step1: decompose the original image $I$ with 4 levels of wavelet, as shown in Figure 1.

Step2: the one dimensional vector $w$ is

$$
w(i)= \begin{cases}-1, & \text { If } w m(i)=0 \\ 1, & \text { If } w m(i)=1\end{cases}
$$

The length of $w$ is $N=p \times q$, then divide $w$ into two weights:

$$
\begin{aligned}
& w_{1}=\{m(i) \mid m(i) \in\{0,1\}, 1 \leq i \leq N / 2\} \\
& w_{2}=\{m(i) \mid m(i) \in\{0,1\}, N / 2<i \leq N\}
\end{aligned}
$$

Step3: work out intensity factor $\mathrm{L}_{2}$ of each coefficient among $I_{2}^{0}$ and $I_{2}^{2}$ on the basis of formula (2) for the two subbands are of the same level. And the intensity factors of the corresponding coefficient are the same too.

$$
L_{2}(2, i, j)=\frac{1}{256} I_{3}^{3}\left(1+\left[\frac{i}{2}\right], 1+\left[\frac{j}{2}\right]\right)
$$

Step4: embed $w_{1}, w_{2}$ to $I_{2}^{0}, I_{2}^{2}$ sub-bands respectively by changing wavelet coefficients.

Take the process of embedding $w_{1}$ as example, select the smallest $N / 2$ numbers of intensity factor to adjust wavelet coefficients using formula (6).

$$
I_{2}^{0^{\prime}}(i, j)=I_{2}^{0}(i, j)+\alpha \times w_{1}(n)
$$

Where $\alpha$ is the regulatory factor and $1 \leq n \leq N / 2$.

Step5: get the watermark contained image $I^{\prime}$ after employ the modified wavelet sub-band to perform reconstruction.

\section{B. Watermark Extraction}

The sub-bands of $I_{2}^{0}$ and $I_{2}^{2}$ enjoy the same method to embed watermark, take $I_{2}^{0}$ as example, extraction of the watermark involves the following steps:

Step1: perform 4 levels of wavelet decomposition on original image $I$ and watermark embedded image $I^{\prime}$.

Step2: calculate intensity factor $L_{2}$ of each coefficient in sub-band of original image $I_{2}^{0}$ on which decide the embedded location of wavelet coefficients. 
Step3: let wavelet coefficient of $I^{\prime}$ subtract the counterpart of $I$ according to the previous obtained location to get the array $M_{1}^{\prime}(n)$ with the length of $N / 2$.

$$
M_{1}^{\prime}(n)=I_{2}^{0}{ }^{\prime}(i, j)-I_{2}^{0}(i, j)
$$

Step4: according to type (8) you can find the embed watermarks $02 \mathrm{I}$.

$$
w_{1}^{\prime}(n)=\left\{\begin{array}{l}
1, \text { if } M_{1}^{\prime}(n) \geq 0 \\
0, \text { if } M_{1}^{\prime}(n) \leq 0
\end{array}\right.
$$

Similarly, you can find the embedded watermark in $I_{2}^{2}$ and get the one-dimensional vector with the length of $N$. It is become possible to get the binary image in the light of the inverse process of watermark construction.

\section{EXPERIMENTAL RESULTS AND PERFORMANCE ANALYSIS}

We assess watermark perceptibility through subject test or quality metrics. Subjective test put up certain value on the final quality evaluation, but is unpractical in the case of research and development.

On this circumstance, the quantization of distortion is more effective, which also make the comparison between different methods tend to be reasonable, because the result do not depend on the subjective assessment. The generally used metrics is the Peak Signal to Noise Ratio (PSNR).

$$
P S N R=10 \lg \left[\frac{M N a_{\max }{ }^{2}}{\sum_{i=0}^{M-1} \sum_{j=0}^{N-1}[f(i, j)-g(i, j)]^{2}}\right]
$$

In the commonly used video sequence and commercial image applications we let $\mathrm{K}=8$ and rightly let $\alpha_{\max }$ be 255 in some documents.

We adopt $N C$ as watermark evaluation criteria.

$$
N C=\frac{\sum_{i} \sum_{j} w(i, j) w^{\prime}(i, j)}{\sum_{i} \sum_{j}[w(i, j)]^{2}}
$$

We perform wavelet decomposition experiment with Haar basis at level 3, select classic $512 \times 512$ "Barbara" as the original image and $32 \times 32$ binary image as the watermark.

In order to balance the imperceptibility and robustness, as document [6] says, select $\alpha=30$ as a regulatory factor shown in Figure 2. Figure 2 (a) is $512 \times 512$ original intensity image, Figure 2 (b) is a $32 \times 32$ watermark image, Figure2 (c) is watermark embedded image (PSNR 40.37dB) and Figure2 (d) is extracted watermark $(N C=1)$.

There are several attack tests and the results below:

(1) JPEG compression. Table I is the comparison of two algorithms under JPEG compression attack, where QF is compression factor and PSNR is the value based on the original image and the JPEG compressed watermark.

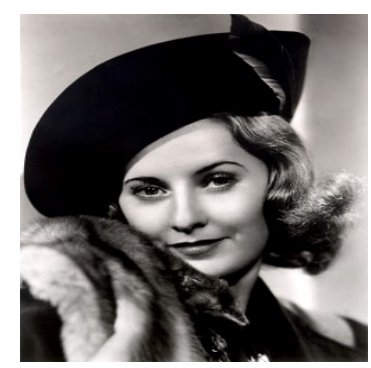

(a) original image

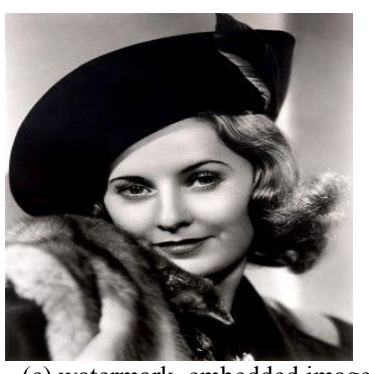

(c) watermark embedded image

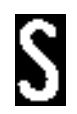

(b) original watermark
Figure 2. Process of watermarking implant and extract

The watermark can be completely extracted when $\mathrm{QF}$ is greater than 13 and is seriously distorted when $\mathrm{QF}=9$, but it would still be able to make out a Chinese character from the

\begin{tabular}{|c|c|c|c|c|c|c|}
\hline \multirow{4}{*}{ I } & $\mathrm{QF}(\%)$ & 93 & 58 & 30 & 14 & 9 \\
\hline & PSNR(dB) & 37.65 & 34.78 & 31.46 & 29.83 & 27.02 \\
\hline & $\begin{array}{c}\text { Extracted } \\
\text { Watermark }\end{array}$ & & & & & \\
\hline & $\mathrm{NC}$ & 1 & 1 & 1 & 1 & 0.92 \\
\hline \multirow{3}{*}{ II } & PSNR(dB) & 37.87 & 34.75 & 31.52 & 29.75 & 27.36 \\
\hline & $\begin{array}{c}\text { Extracted } \\
\text { Watermark }\end{array}$ & & & & & \\
\hline & $\mathrm{NC}$ & 1 & 1 & 1 & 1 & 0.95 \\
\hline
\end{tabular}
watermark image.

TABLE I. COMPARISON OF TWO ALGORITHM UNDER JPEG COMPRESSED ATTACK

I, II refer to algorithms in this paper and document [10] respectively

TABLE II. COMPARISON OF TWO ALGORITHM AFTER ADDING GAUSSIAN NOISE

\begin{tabular}{|c|c|c|c|c|c|}
\hline \multirow{4}{*}{ I } & Density & 10 & 30 & 50 & 80 \\
\cline { 2 - 6 } & PSNR(dB) & 32.77 & 22.23 & 17.98 & 13.45 \\
\cline { 2 - 6 } & $\begin{array}{c}\text { Extracted } \\
\text { Watermark }\end{array}$ & & & & \\
\cline { 2 - 6 } & NC & 1 & 1 & 0.97 & 0.92 \\
\hline \multirow{4}{*}{ II } & PSNR(dB) & 32.72 & 22.24 & 17.92 & 13.42 \\
\cline { 2 - 6 } & Extracted & & & & \\
& Watermark & & & & \\
\cline { 2 - 6 } & NC & 1 & 1 & 0.98 & 0.91 \\
\hline
\end{tabular}

I, II refer to algorithms in this paper and document [10] respectively

(2) Add Gaussian noise. 'Density' stands for density factor range from 1 to 100 in Table II, experimental results 
demonstrate that this algorithm with strong robustness to resist Gaussian noise.

(3) Contrast enhancement. Table III shows that the watermark can still be extracted when gray value of each element in watermark embedded image plus 10, 20, 40, 60 respectively.

TABLE III. $\quad$ COMPARISON OF TWO ALGORITHM AFTER CONTRAST ADJUSTED

\begin{tabular}{|c|c|c|c|c|c|}
\hline \multirow{4}{*}{ I } & Contrast & 10 & 20 & 40 & 60 \\
\cline { 2 - 6 } & PSNR(dB) & 27.65 & 22.38 & 16.07 & 10.95 \\
\cline { 2 - 6 } & $\begin{array}{c}\text { Extracted } \\
\text { Watermark }\end{array}$ & & & & \\
\cline { 2 - 6 } & NC & 1 & 1 & 1 & 0.93 \\
\hline \multirow{4}{*}{ II } & PSNR(dB) & 27.89 & 23.29 & 16.36 & 11.37 \\
\cline { 2 - 6 } & $\begin{array}{c}\text { Extracted } \\
\text { Watermark }\end{array}$ & & & & \\
\cline { 2 - 6 } & NC & 1 & 1 & 1 & 0.94 \\
\hline
\end{tabular}

I, II refer to algorithms in this paper and document [10] respectively

\section{CONCLUSIONS}

We propose a wavelet domain watermarking algorithm based on human visual system in the article. The results of experiment indicate that the watermark embedded image has better imperceptibility which attribute to application of human vision model. Attack tests confirmed that this algorithm is with strong robustness to several conventional watermarking attacks such as JPEG compression, adding Gaussian noise, median filter and contrast enhancement. The selection of wavelet coefficients only utilizes visual sensitivity of noise in different intensity domains, the application of visual model could continue to improve. And how to combine with the visual sensitivity that human eye on noise of image textural area will be the direction of research in the future.

\section{ACKNOWLEDGMENT}

This work is supported by Project of SiChuan Educational Hall with grant name "research on multimode medical image fusion”.

\section{REFERENCES}

[1] H. Li, G. X. Zhu and Y. T. zhu, "A visual model weighted image watermarking method using wavelet decomposition", Joural of China Institute of Communications, vol 21, Jun. 2000, pp. 37-41

[2] H. LI, G. X. Zhu and Y. T. Zhu, "A Visual Model Weighted Cosine Transform for Hiding Watermark in Images", Acta Electronica Sinica, vol 28, Oct. 2000, pp. 110-113.

[3] R. B. Wolfgang, C. I. Podilchuk and E. J. Delp. "Perceptual Watermarks for Digtal Image and Video", Proc. IEEE, Jan. 2008, pp. 1108-1126.

[4] B. Meng, L. Zhou and W. J. Zhu e.t., "An Image Digital Watermarking Algorithm Based on Wavelet Transform", Jouranl of Computer Aided Design \& Computer Graphics, vol 12, Oct. 2000, pp. $752-755$.

[5] X. Feng, X. S. Zhu and Z. Tang, "Progress of research on digital watermarking techniques applied to multimedia", Computer Engineering and Applications, vol 43, 2007, pp. 1- 6.

[6] Q. M. Peng, X. F. Yang and S. Huan etc., "A Novel Adaptive Blind Digital Watermarking Algorithm”, Computer Science, vol 32, Aug. 2005, pp: 63-66.

[7] L. Xiao, Z. H. Wei, H. Z. Wu. "A digital watermarking in wavelet domain utilizing human visual masking", Journal of China Institute of Communications, vol 23, Mar. 2002, pp. 100-106.

[8] A. S. Lewis and G. Knowles, "Image Compression Using the 2-D Wavelet Transform". IEEE Trans. on Image Processing, vol 1, Feb. 1992, pp. 244-250.

[9] M. Barni, F. Bartolini and A. Piva, "Improved Wavelet-based Watermarking Through Pixel-wise Masking", IEEE Trans. on Image Processing, vol. 10, May. 2001, pp. 783-791.

[10] Z. F. Wang, B. C. Shi and N. C. Wang, "Robust watermarking algorithm using wavelet transform and HVS", Journal of Huazhong University of Science and Technology(Nature Science Edition), vol. 43, Jan. 2007, pp. 31-33. 